\title{
Case Report: Anti-MOG Antibody Seroconversion Accompanied by Dimethyl Fumarate Treatment
}

\author{
Keita Takahashi ${ }^{1}$, Hideyuki Takeuchi ${ }^{1 *}$, Ryoko Fukai ${ }^{1}$, Haruko Nakamura ${ }^{1}$, \\ Keisuke Morihara ${ }^{1}$, Yuichi Higashiyama ${ }^{1}$, Toshiyuki Takahashi ${ }^{2}$, Hiroshi Doi $^{1}$ and \\ Fumiaki Tanaka ${ }^{1 *}$ \\ ${ }^{1}$ Department of Neurology and Stroke Medicine, Yokohama City University Graduate School of Medicine, Yokohama, Japan, \\ ${ }^{2}$ Department of Neurology, Tohoku University Graduate School of Medicine, Sendai, Japan
}

\section{OPEN ACCESS}

Edited by:

Pamela Ann McCombe, The University of

Queensland, Australia

Reviewed by:

Giacomo Casella,

Thomas Jefferson University,

United States

Alice Mariottini,

University of Florence, Italy

*Correspondence:

Hideyuki Takeuchi

htake@yokohama-cu.ac.jp

Fumiaki Tanaka

ftanaka@yokohama-cu.ac.jp

Specialty section

This article was submitted to

Multiple Sclerosis and

Neuroimmunology,

a section of the journal

Frontiers in Immunology

Received: 03 November 2020

Accepted: 27 January 2021

Published: 15 February 2021

Citation:

Takahashi K, Takeuchi H, Fukai R,

Nakamura $H$, Morihara $K$,

Higashiyama Y, Takahashi T, Doi H

and Tanaka F (2021) Case Report:

Anti-MOG Antibody Seroconversion

Accompanied by Dimethyl Fumarate

Treatment.

Front. Immunol. 12:625465

doi: 10.3389/fimmu.2021.625465
Here we report three cases of anti-myelin oligodendrocyte glycoprotein (MOG) antibody-associated disease (MOGAD) mimicking multiple sclerosis in which seropositivity for anti-MOG antibodies occurred during disease-modifying drug dimethyl fumarate (DMF) treatment. These patients developed relapses with anti-MOG antibody seroconversion after switching from fingolimod or steroid pulse therapy to DMF, which was associated with peripheral lymphocyte recovery. MOGAD is considered a humoral immune disease, and DMF reportedly enhances Th2-skewed humoral immune activity. Therefore, we suggest that DMF, but not fingolimod, may exacerbate humoral immune imbalance and enhance autoantibody production, leading to aggravation of MOGAD.

Keywords: anti-myelin oligodendrocyte glycoprotein antibody-associated disease, dimethyl fumarate, fingolimod, multiple sclerosis, seroconversion

\section{INTRODUCTION}

Anti-myelin oligodendrocyte glycoprotein (MOG) antibody-associated disease (MOGAD), which makes up $\sim 40 \%$ of anti-aquaporin 4 (AQP4) antibody-negative neuromyelitis optica spectrum disorders, is often difficult to distinguish from multiple sclerosis (MS) (1). Whether diseasemodifying drugs (DMDs) widely used in MS are also effective in MOGAD remains unclear. Here we report three cases of MOGAD treated as atypical MS due to negativity for specific antibodies in which seroconversion for anti-MOG antibodies occurred when DMD was switched to dimethyl fumarate (DMF).

\section{CASE REPORT}

The first patient was a 27-year-old woman who experienced two attacks of optic neuritis and transverse myelitis with short cervical and thoracic cord lesions in 4 years (Figure 1A). She experienced severe attacks of left retrobulbar neuritis and transverse myelitis at the age of 24. Magnetic resonance imaging (MRI) showed T2-hyperintense lesions in the left optic nerve (Figure 1B) and left dorsal horn and fasciculus at the C3 level (Figures 1C,D). Optical coherence tomography (OCT) detected a significant decrease in retinal nerve fiber layer (RNFL) thickness in the left eye (Figure 1E). Cerebrospinal fluid (CSF) analysis revealed a slight elevation in protein levels and increases in myelin basic protein (MBP) levels and IgG index, but not in cell count or oligoclonal bands (Table 1; Patient 1, pre). Neither anti-AQP4 nor anti-MOG antibodies were detected in serum samples using an internationally standardized cell-based assay (CBA) (2-4). She 

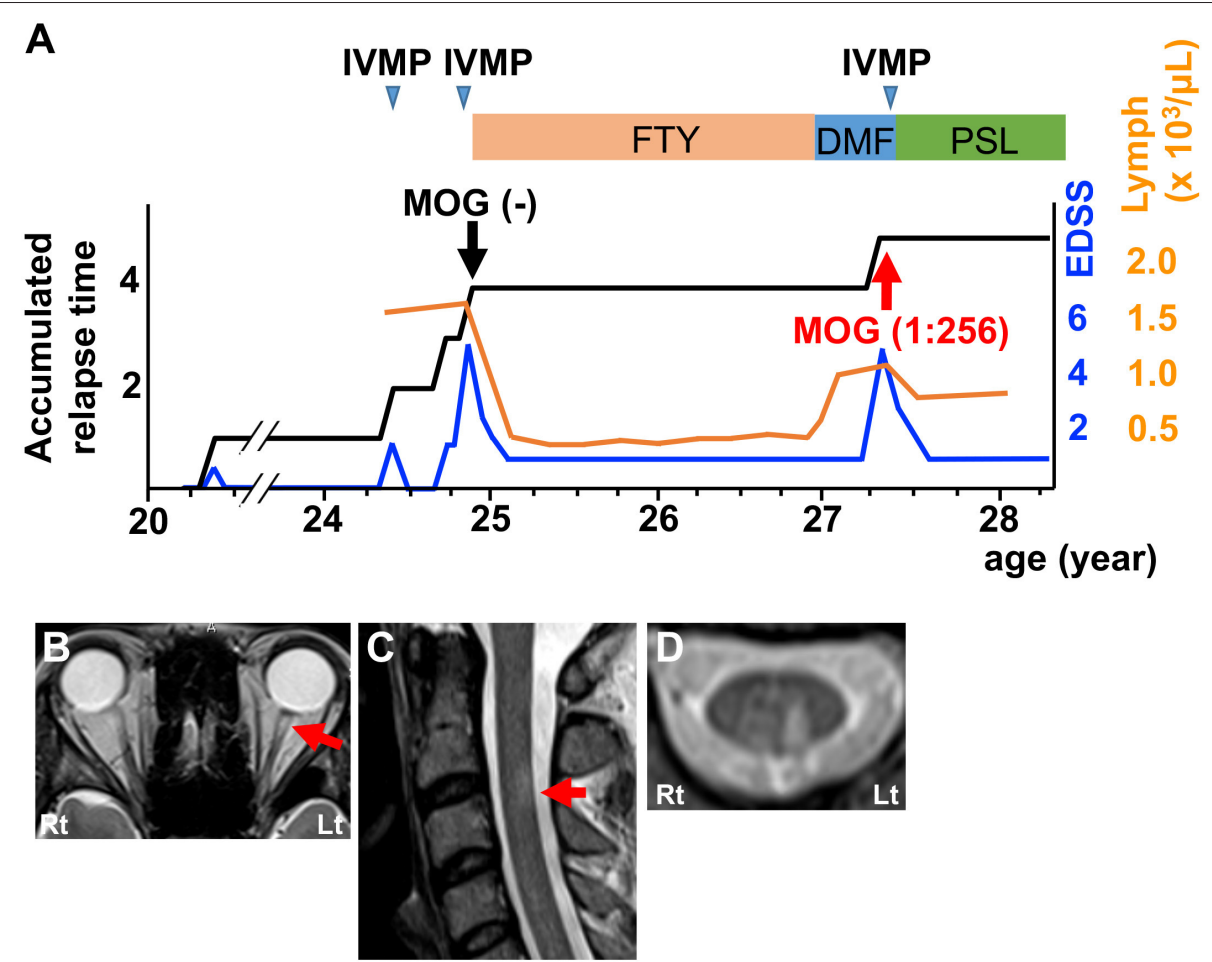

E
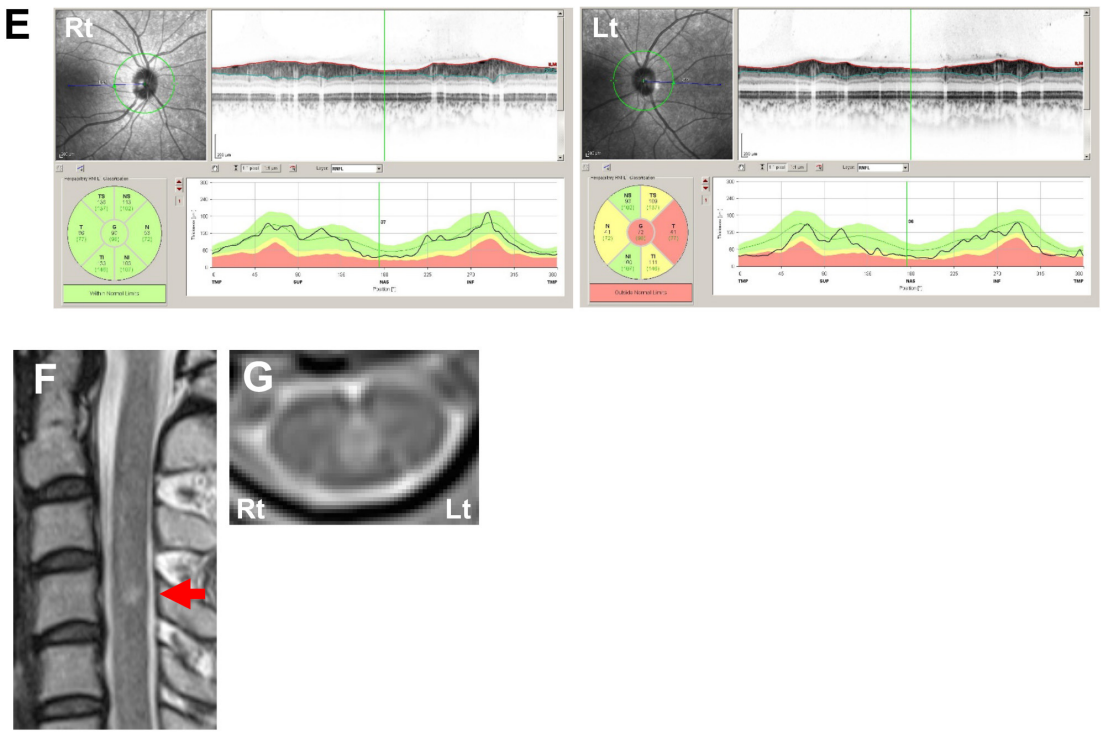

FIGURE 1 | Clinical features of Patient 1. (A) Graph of the time course for relapses and treatments. (B) Axial T2-weighted orbit MRI showing left optic nerve lesion (red arrow). (C) Sagittal T2-weighted cervical MRI showing a short cervical lesion at the C3 level (red arrow). (D) Axial T2-weighted cervical MRI showing a lesion in the left dorsal horn and fasciculus at the C3 level. (E) OCT showing significant RNFL thinning in the left eye. Green, normal RNFL thickness (5th-95th percentile); yellow, mild-to-moderate RNFL thinning (1st-5th percentile); red, severe RNFL thinning ( $<1$ st percentile). (F) Sagittal T2-weighted cervical MRI showing a short cervical lesion at the C4 level (red arrow). (G) Axial T2-weighted cervical MRI showing a lesion around the central canal at the C4 level. DMF, dimethyl fumarate; FTY, fingolimod; IVMP, intravenous methylprednisolone pulse therapy; PSL, prednisolone; EDSS, Expanded Disability Status Scale score; Lymph, peripheral lymphocyte count.

had been effectively treated with fingolimod for the diagnosis of atypical MS for 2.5 years. Since she desired to have a child, fingolimod was switched to DMF. Three months later, she developed a severe relapse of transverse myelitis with a short cervical cord lesion accompanied by recovery from lymphocytopenia (Figure 1A). MRI showed T2-hyperintense lesion around the central canal at the C4 level (Figures 1F,G). CSF analysis also indicated elevation of protein levels, MBP levels, and IgG index, but pleocytosis and oligoclonal bands were not detected (Table 1; Patient 1, post). At that time, the second 
CBA using the same methodology (3) revealed seroconversion for anti-MOG antibodies (titer, 1:256; cut-off, $\geq 1: 128$ ). We reconfirmed that the frozen serum sample from the time of

TABLE 1 | CSF findings of three patients.

\begin{tabular}{lccc}
\hline & $\begin{array}{c}\text { Patient 1 } \\
\text { pre/post }\end{array}$ & $\begin{array}{c}\text { Patient 2 } \\
\text { pre/post }\end{array}$ & $\begin{array}{c}\text { Patient 3 } \\
\text { pre/post }\end{array}$ \\
\hline Cell count (cells/ $\mu \mathrm{l})$ & $2 / 2$ & $20 / 10$ & $2 / 1$ \\
Protein $(\mathrm{mg} / \mathrm{dl})$ & $44 / 86$ & $51 / 31$ & $31 / 31$ \\
Myelin basic protein $(\mathrm{pg} / \mathrm{ml})$ & $86.5 / 103.1$ & $\mathrm{ND} / \mathrm{ND}$ & $1729.8 / \mathrm{ND}$ \\
IgG index & $0.91 / 0.64$ & $2.06 / 1.36$ & $0.36 / 0.41$ \\
Oligoclonal band & $-/-$ & $+/+$ & $-/-$ \\
\hline
\end{tabular}

Pre, CSF collection before seroconversion for anti-MOG antibodies; post, CSF collection after seroconversion for anti-MOG antibodies; ND, not detected. the first CBA was truly seronegative for anti-MOG antibodies. Intravenous methylprednisolone pulse therapy $(1 \mathrm{~g} /$ day for 3 consecutive days; IVMP) markedly decreased symptoms. She has had no relapses with subsequent oral prednisolone therapy (20 mg/day) for over 1 year (Figure 1A).

The second patient was a 27 -year-old woman who had several attacks of optic neuritis and transverse myelitis involving short lesions in the cervical cord in a year (Figure 2A). She experienced a severe attack of optic neuritis around the age of 25 , as shown on fluid-attenuated inversion recovery (FLAIR) MRI (Figure 2B). OCT revealed severe RNFL thinning in the right eye and mild RNFL thinning in the left eye (Figure 2C). CSF analysis detected pleocytosis, elevations in protein levels and IgG index, and the presence of oligoclonal bands, but not MBP (Table 1; Patient 2, pre). A CBA did not detect anti-AQP4 or anti-MOG antibodies in the serum. She had been treated with interferon- $\beta$ followed by fingolimod for the diagnosis of

\section{A}
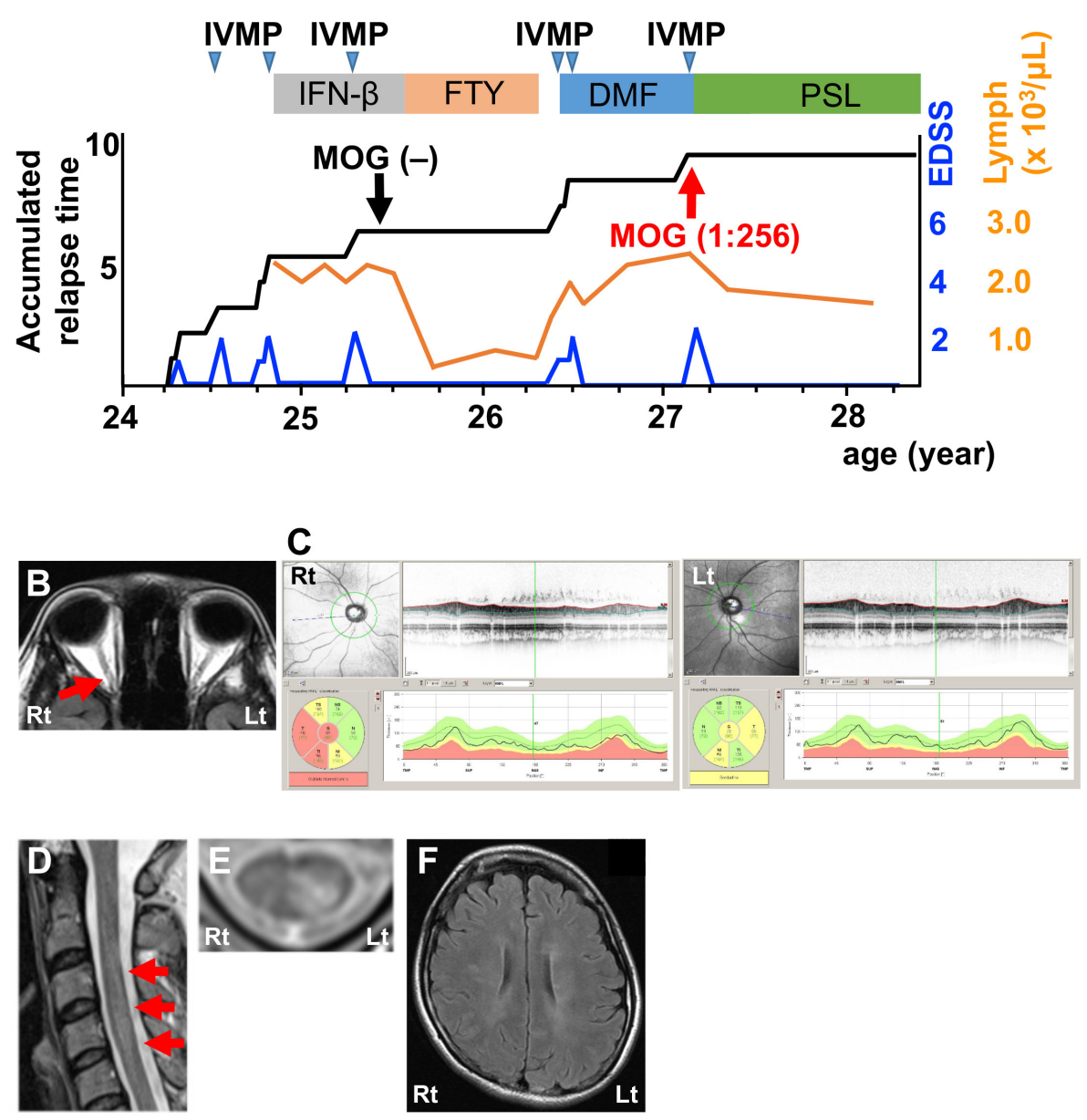

FIGURE 2 | Clinical features of Patient 2. (A) Graph of the time course for relapses and treatments. (B) Axial FLAIR orbit MRI showing right optic nerve lesions (red arrow). (C) OCT showing severe RNFL thinning in the right eye and mild RNFL thinning in the left eye. Green, normal RNFL thickness (5th-95th percentile); yellow, mild-to-moderate RNFL thinning (1st-5th percentile); red, severe RNFL thinning ( $<1$ st percentile). (D) Sagittal T2-weighted cervical MRI showing a lesion at the C3-4 levels (red arrows). (E) Axial T2-weighted cervical MRI showing lesions in the left dorsal horn and fasciculus at the C4 level. (F) Axial FLAIR brain MRI showing periventricular and subcortical lesions. DMF, dimethyl fumarate; FTY, fingolimod; IFN- $\beta$, interferon- $\beta$; IVMP, intravenous methylprednisolone pulse therapy; PSL, prednisolone; EDSS, Expanded Disability Status Scale score; Lymph, peripheral lymphocyte count. 

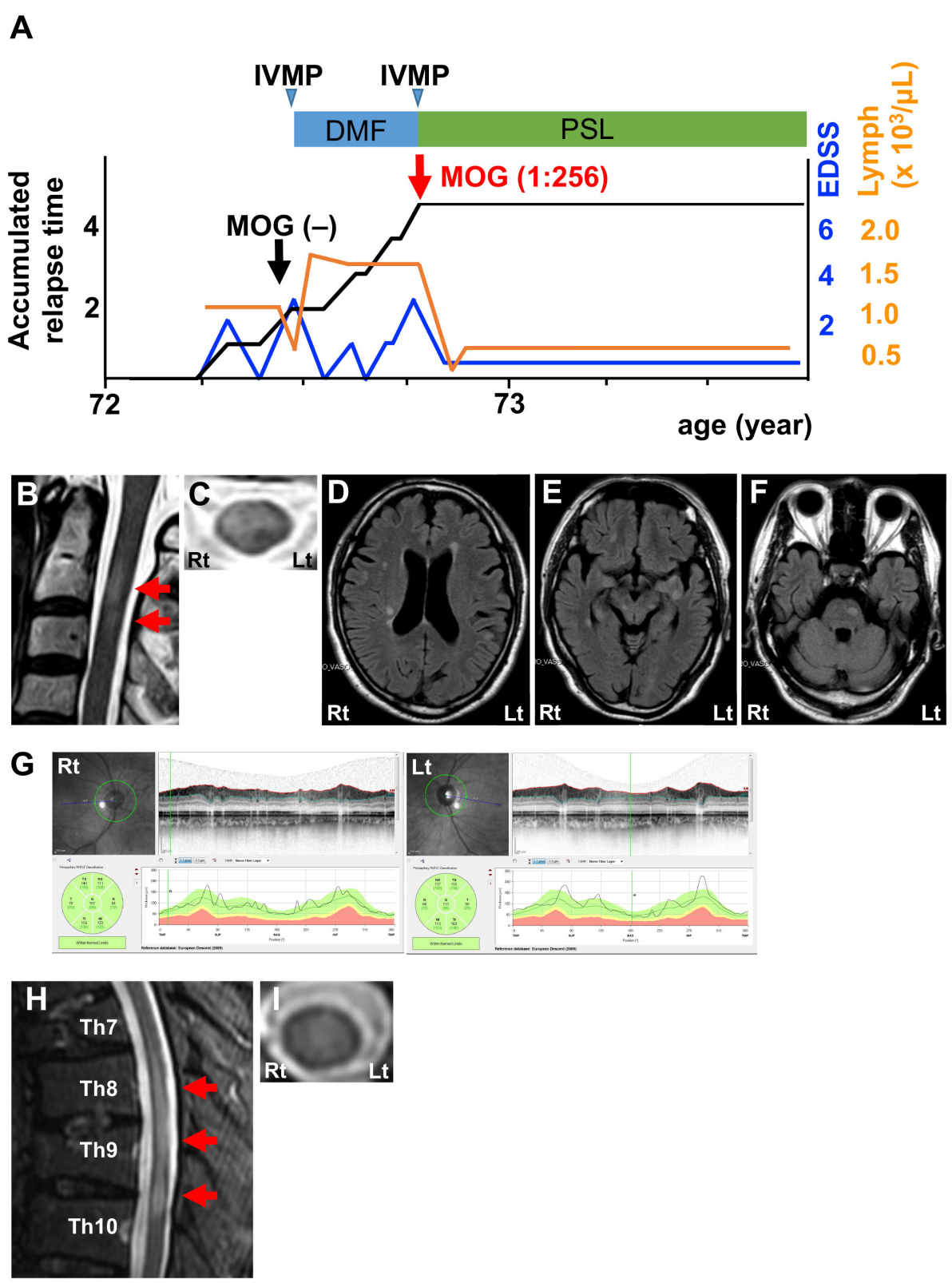

FIGURE 3 | Clinical features of Patient 3. (A) Graph of the time course for relapses and treatments. (B) Sagittal T2-weighted cervical MRI showing short lesions at the C2-3 level (red arrows). (C) Axial T2-weighted cervical MRI showing a ventral-dominant lesion at the C2 level. (D-F) Axial FLAIR brain MRI showing lesions in the periventricular area, subcortical area, and brain stem. (G) OCT showing normal RNFL thickness. Green, normal RNFL thickness (5th-95th percentile). (H) Sagittal T2-weighted thoracic MRI showing lesions at the Th8-10 levels (red arrows). (I) Axial T2-weighted cervical MRI showing lesion at the Th8 level around the central canal. DMF, dimethyl fumarate; IVMP, intravenous methylprednisolone pulse therapy; PSL, prednisolone; EDSS, Expanded Disability Status Scale score; Lymph, peripheral lymphocyte count.

atypical MS for 1 year without any relapses, but DMD was changed to DMF because of epidural hematoma as an unexpected adverse side effect of fingolimod (5). After 1 year, she had a relapse of transverse myelitis with a short cervical cord lesion (Figures 2D,E) and asymptomatic MS-like supratentorial lesions (Figure 2F) following lymphocyte recovery (Figure 2A). CSF analysis revealed a profile that was similar to the profile before DMF treatment, except for normal protein levels at this time
(Table 1; Patient 2, post). This patient also had seroconversion for anti-MOG antibodies (titer, 1:256) using the same methodology. Serum from the time of the first CBA was reconfirmed to be truly seronegative. IVMP substantially decreased her symptoms. Oral prednisolone (20 mg/day) completely suppressed the relapse for more than a year (Figure 2A).

The third patient was a 73-year-old man with a severe attack of transverse myelitis in the previous year (Figure 3A). FLAIR 
MRI showed short lesions in the cervical cord (Figures 3B,C) as well as asymptomatic lesions in the periventricular area, subcortical area, and brain stem (Figures 3D-F). OCT indicated no signs of optic neuritis (Figure 3G). CSF analysis revealed a prominent increase in $\mathrm{MBP}$ levels, but was otherwise normal (Table 1; Patient 3, pre). Neither anti-AQP4 nor anti-MOG antibodies were detected in the serum using the same CBA described for the other 2 patients. IVMP effectively resolved his symptoms. He was subsequently treated with DMF for the diagnosis of atypical MS. Lymphocyte counts became higher than pre-treatment levels and reached a plateau (Figure 3A). Three months later, he developed a severe relapse of transverse myelitis. MRI revealed a FLAIR-hyperintense lesion around the central canal extending from the Th8 level to the Th10 level (Figures 3H,I). CSF analysis showed a normal profile, including normal MBP levels (Table 1; Patient 3, post). A CBA for antiMOG antibodies turned positive at that time (titer, 1:256). We also reconfirmed seronegativity for anti-MOG antibodies in a frozen serum sample from the time of the first CBA. IVMP was effective in decreasing his symptoms again. To date, he has been in remission with oral prednisolone therapy $(20 \mathrm{mg} /$ day $)$ for more than a year (Figure $\mathbf{3 A}$ ).

\section{DISCUSSION}

Although therapy for MOGAD is yet to be established, according to consensus among international experts $(6,7)$, immunosuppressive therapy (e.g., corticosteroids, azathioprine, tacrolimus, mycophenolate mofetil, and methotrexate) is the mainstay for treatment to prevent relapse. In particular, B cell depleting therapies such as rituximab have shown good therapeutic responses, but relapse occurs immediately after B cell recovery. These data strongly suggest that suppression of humoral immunity is likely the key therapeutic strategy for MOGAD. DMDs for MS such as interferon$\beta$, glatiramer acetate, and natalizumab might not be efficacious, but the effectiveness of fingolimod remains uncertain $(6,7)$. Although a recent report mentioned that DMF is ineffective but not harmful in a patient with MOGAD (8), the effectiveness of DMF has yet to be determined.

Initially, our patients were diagnosed with atypical MS because they were seronegative for specific antibodies and had good response to IVMP and fingolimod. However, seroconversion for anti-MOG antibodies occurred when DMD therapy was switched to DMF. We propose two possible mechanisms to explain this seroconversion. One possibility is that the first CBA results in all patients were falsenegative due to fluctuations in autoantibody titers, and the timing of seroconversion and switching to DMF was just a coincidence because the titers from the second CBA in all patients were relatively low (1:256). Despite low titers of antiMOG antibodies, all patients have been in remission with oral prednisolone therapy for at least 1 year after their last relapse. This good response to corticosteroid therapy suggests that these patients truly had MOGAD. Another possibility is that DMF treatment enhanced anti-MOG autoantibody production whereas fingolimod treatment effectively suppressed it. The main therapeutic effect of fingolimod is immunosuppression due to the retention of auto-reactive lymphocytes within secondary lymphoid organs, whereas the net activity of DMF is mainly a pro-tolerogenic lymphocyte shift (9). As humoral immunosuppression, fingolimod is likely efficacious in MOGAD whereas DMF is not efficacious. A previous report documented a similar seroconversion for anti-MOG antibodies during interferon- $\beta$ treatment, which modulates immune balance from Th1/Th17-mediated cellular immunity to Th2-mediated humoral immunity and may promote autoantibody synthesis (10). Like interferon- $\beta$, DMF also enhances Th2-skewed humoral immune activity (11). Another report demonstrated that DMF induces severe relapses of neuromyelitis optica spectrum disorders, indicating that DMF has a detrimental effect in humoral immunity-mediated diseases (12). In fact, our cases revealed that fingolimod effectively suppresses peripheral lymphocyte count and switching from fingolimod to DMF or initiating DMF leads to a recovery of peripheral lymphocyte count accompanied by seroconversion for anti-MOG antibodies (Figures 1A, 2A, 3A). These findings suggest that DMF might exacerbate Th2-prone humoral autoimmunity. In addition, two of three patients (Patients 1 and 2) had similar or higher peripheral lymphocyte counts at the time of the first CBA than at the time of the second CBA, suggesting that the Th2-skewed peripheral lymphocyte subpopulation shift associated with DMF (11) may affect MOGAD development rather than the higher absolute number of peripheral lymphocytes.

In conclusion, we presented three cases of MOGAD with seroconversion for anti-MOG antibodies accompanied by recurrence of clinical disability after treatment with DMF. Thus, DMF should be avoided in MOGAD, whereas fingolimod may be efficacious because it suppresses humoral immunity.

\section{PRACTICAL IMPLICATIONS}

Dimethyl fumarate may exacerbate anti-myelin oligodendrocyte glycoprotein antibody-associated disease whereas fingolimod may be effective against this disease.

\section{DATA AVAILABILITY STATEMENT}

The original contributions presented in the study are included in the article/supplementary material, further inquiries can be directed to the corresponding authors.

\section{ETHICS STATEMENT}

Written informed consent was obtained from the patients for the publication of this case series in accordance with the Declaration of Helsinki. 


\section{AUTHOR CONTRIBUTIONS}

KT, HT, RF, HN, KM, YH, and HD examined and treated the patient. TT assessed anti-MOG antibody titers. KT and HT analyzed data. HT and FT designed and supervised this study. KT, HT, and FT wrote the manuscript. All authors contributed to the article and approved the submitted version.

\section{REFERENCES}

1. Narayan R, Simpson A, Fritsche K, Salama S, Pardo S, Mealy M, et al. MOG antibody disease: a review of MOG antibody seropositive neuromyelitis optica spectrum disorder. Mult Scler Relat Disord. (2018) 25:66-72. doi: 10.1016/j.msard.2018.07.025

2. Takahashi T, Fujihara K, Nakashima I, Misu T, Miyazawa I, Nakamura M, et al. Anti-aquaporin-4 antibody is involved in the pathogenesis of NMO: a study on antibody titre. Brain. (2007) 130:1235-43. doi: 10.1093/brain/awm062

3. Sato DK, Callegaro D, Lana-Peixoto MA, Waters PJ, de Haidar Jorge FM, Takahashi T, et al. Distinction between MOG antibody-positive and AQP4 antibody-positive NMO spectrum disorders. Neurology. (2014) 82:47481. doi: 10.1212/WNL.0000000000000101

4. Waters P, Woodhall M, O'Connor KC, Reindl M, Lang B, Sato DK, et al. MOG cell-based assay detects non-MS patients with inflammatory neurologic disease. Neurol Neuroimmunol Neuroinflamm. (2015) 2:e89. doi: 10.1212/NXI.0000000000000089

5. Fukai R, Takahashi K, Abe H, Higashiyama Y, Doi H, Takeuchi H, et al. Non-traumatic acute epidural hematoma in multiple sclerosis treated with fingolimod. Front Neurol. (2019) 10:763. doi: 10.3389/fneur.2019.00763

6. Fujihara K, Sato DK, Nakashima I, Takahashi T, Kaneko K, Ogawa $\mathrm{R}$, et al. Myelin oligodendrocyte glycoprotein immunoglobulin Gassociated disease: an overview. Clin Exp Neuroimmunol. (2018) 9:48-55. doi: 10.1111/cen3.12434

7. Reindl M, Waters P. Myelin oligodendrocyte glycoprotein antibodies in neurological disease. Nat Rev Neurol. (2019) 15:89-102. doi: 10.1038/s41582-018-0112-x

8. Warabi Y, Takahashi T, Isozaki E. Dimethyl fumarate was ineffective but not harmful for a patient with myelin oligodendrocyte

\section{FUNDING}

This study was supported partly by Grants-in-Aid for Scientific Research from the Ministry of Education, Culture, Sports, Science and Technology of Japan, grants from the Ministry of Health, Labor and Welfare of Japan, and a grant for Strategic Research Promotion from Yokohama City University.

glycoprotein antibody disease. Cureus. (2019) 11:e6040. doi: 10.7759/ cureus. 6040

9. Rommer PS, Milo R, Han MH, Satyanarayan S, Sellner J, Hauer L, et al. Immunological aspects of approved MS therapeutics. Front Immunol. (2019) 10:1564. doi: 10.3389/fimmu.2019.01564

10. Pawlitzki M, Campe C, Rolfes L, Heinze HJ, Leypoldt F, Wandinger $\mathrm{KP}$, et al. Transient MOG antibody seroconversion associated with immunomodulating therapy. Mult Scler Relat Disord. (2020) 37:101420. doi: 10.1016/j.msard.2019.101420

11. Kira JI, Unexpected exacerbations following initiation of disease-modifying drugs in neuromyelitis optica spectrum disorder: Which factor is responsible, anti-aquaporin 4 antibodies, B cells, Th1 cells, Th2 cells, Th17 cells, or others? Mult Scler. (2017) 23:1300-2. doi: 10.1177/1352458517703803

12. Yamout BI, Beaini S, Zeineddine MM, Akkawi N. Catastrophic relapses following initiation of dimethyl fumarate in two patients with neuromyelitis optica spectrum disorder. Mult Scler. (2017) 23:1297-300. doi: 10.1177/1352458517694086

Conflict of Interest: The authors declare that the research was conducted in the absence of any commercial or financial relationships that could be construed as a potential conflict of interest.

Copyright (c) 2021 Takahashi, Takeuchi, Fukai, Nakamura, Morihara, Higashiyama, Takahashi, Doi and Tanaka. This is an open-access article distributed under the terms of the Creative Commons Attribution License (CC BY). The use, distribution or reproduction in other forums is permitted, provided the original author(s) and the copyright owner(s) are credited and that the original publication in this journal is cited, in accordance with accepted academic practice. No use, distribution or reproduction is permitted which does not comply with these terms. 